1 Universidade Federal de Santa Catarina (UFSC), Programa de Pós-Graduação em Saúde Coletiva - Florianópolis (SC), Brasil.

elismonteiro1@yahoo.com.br

2 Universidade Federal de Santa Catarina (UFSC), Programa de Pós-Graduação em Saúde Coletiva - Florianópolis (SC), Brasil.

jtelino@gmail.com

\section{Promoção do uso racional de medicamentos: uma proposta de modelo avaliativo da gestão municipal}

\author{
Promoting the rational use of drugs: a proposal for an evaluation \\ model of municipal management
}

Elis Roberta Monteiro', Josimari Telino de Lacerda²

RESUMO O artigo propõe um modelo avaliativo da gestão municipal na promoção do uso racional de medicamentos. Análises documentais, revisão de literatura e reuniões com grupos de interessados orientaram a elaboração do modelo. Para a sua validação, utilizou-se a técnica de conferência de consenso. A matriz é composta por 28 indicadores agregados nas dimensões estruturais e operacionais. $\mathrm{O}$ estudo possibilitou a explicitação e o entendimento dos objetivos e metas da intervenção, indicando a possibilidade de o modelo expressar a atuação da gestão na promoção do uso racional de medicamentos. E concluiu, pela plausibilidade do modelo avaliativo, a sua pertinência em estudos dessa natureza.

PALAVRAS-CHAVE Avaliação em saúde. Gestão em saúde. Uso de medicamentos.

ABSTRACT This paper proposes an evaluation model of municipal management in promoting the rational use of drugs. Documental analysis, literature review and meetings with stakeholder groups have guided the development of models. For the validation, the consensus conference technique was used. This matrix is composed of 28 indicators aggregated in structural and operational dimensions. This study enabled the clarification and understanding of this intervention objectives and goals, indicating the possibility of the model to express management performance in promoting rational use of drugs. It has concluded, by the plausibility of assessment model, its relevance in studies of this nature.

KEYWORDS Health evaluation. Health management. Drug utilization. 


\section{Introdução}

Os medicamentos têm papel central na terapêutica contemporânea, com potencial de aliviar sintomas e, em alguns casos, curar doenças. O acesso a eles é considerado um direito humano fundamental, porém, mundialmente, os medicamentos estão incorporados à lógica capitalista de consumo. A forte pressão para a uniformização do comportamento humano, influenciado pelo modelo biomédico, e as estratégias de vendas da indústria farmacêutica, incentivam o uso inadequado e intensificam o processo de medicalização da sociedade. Este tema é discutido entre as autoridades mundiais há vários anos e é considerado um problema global e extremamente sério (CAPONI ET AL., 2010; WHO, 2002).

A situação brasileira na utilização de medicamentos é igualmente preocupante. O País apresenta alta prevalência de consumo de medicamentos e baixa adesão da população à prescrição, conforme a orientação dos serviços. Este problema é agravado pelo processo da automedicação, muito presente na dinâmica social do Brasil (LEITE; VIEIRA; VEBER, 2008).

A Constituição Federal brasileira de 1988 atribui ao Estado a responsabilidade de garantir saúde à população, através do acesso a bens e serviços, além da adoção de medidas de redução de riscos a doenças e agravos. $\mathrm{Na}$ Política Nacional de Medicamentos (PNM), os gestores são responsáveis pela Promoção do Uso Racional de Medicamentos (Purm) (BRASIL, 1988, 1998).

No Brasil,o Uso Racionalde Medicamentos (URM) foi definido como o processo que compreende prescrição apropriada, disponibilidade oportuna, dispensação adequada e consumo em doses, intervalos e períodos de tempo indicados de medicamentos eficazes, seguros e de qualidade (BRASIL, 1998).

Por esse conceito, o URM resulta de relações entre diferentes atores sociais, evidenciando as responsabilidades do governo, dos profissionais de saúde e da sociedade para a sua efetivação (COELHO; PINHEIRO; MAGARINOSTORRES, 2014). No nível municipal, cabe ao gestor disponibilizar os recursos e subsídios necessários para a implementação e a efetivação do uso mais adequado dos medicamentos, na sua área de atuação.

Os medicamentos representam boa parcela dos gastos públicos e não são substâncias inócuas. Promover o uso adequado auxilia a racionalização de recursos e amplia a qualidade dos tratamentos em saúde (MELO; RIBEIRO; STORPIRTIS, 2006; COELHO; PINHEIRO; MAGARINOS-TORRES, 2014). Conhecer os mecanismos que auxiliam a Purm é fundamental e, nesse contexto, os estudos de avaliação apresentam-se como uma ferramenta para a obtenção de conhecimento, e norteadora de mudanças.

Avaliar consiste em fazer um julgamento de valor sobre uma intervenção, com o objetivo de colaborar na tomada de decisões. Trata-se de um instrumento útil, que tem como objetivo fundamental a identificação de problemas e a reorientação de ações e serviços (PINTO JÚNIOR ET AL., 2015).

A ampla discussão sobre o URM em nível mundial, o preocupante quadro brasileiro na utilização de medicamentos, as consequências e o desperdício de recursos devido ao uso inadequado, a relevância das ações da gestão na Purm, o tempo de implantação da PNM e a importância de estudos avaliativos para tomada de decisão tornam oportuna a proposição de um modelo avaliativo da gestão municipal na Purm. Busca-se uma melhor compreensão das responsabilidades do gestor municipal na Purm para subsidiar o planejamento e a implementação de ações, organização e reorientação dessa intervenção nos municípios.

Este artigo tem o objetivo de apresentar uma proposta de modelo avaliativo da gestão municipal na Purm, composto por: modelo teórico, modelo lógico, matriz de análise e julgamento e recomendações necessárias para futuros estudos avaliativos. 


\section{Métodos}

Trata-se de um estudo baseado na metodologia Framework for Program Evaluation in Public Health, desenvolvida pelo Center for Disease Control and Prevetion (CDC, 1999) e revista por Leviton e colaboradores (2010). Trata-se de um estudo de abordagem qualitativa, realizado entre os períodos de março de 2014 e julho de 2015, que foi composto pelas seguintes etapas: formação de um grupo de interessados; descrição e entendimento da intervenção; focalização do objeto; identificação e discussão dos elementos de análise e fontes de evidências; justificativa das conclusões e compartilhamento das lições aprendidas. Durante a execução das etapas da pesquisa, foram realizadas oficinas de discussões com os participantes para assegurar a plausibilidade dos elementos incluídos nos modelos teórico e lógico e na matriz de análise e julgamento (CDC, 1999; LEVITON ET AL., 2010).

As ações da gestão municipal que propiciam o URM foram consideradas, neste estudo, como uma intervenção constituída por um conjunto de meios físicos, humanos, financeiros e simbólicos, organizados em um contexto político, com o objetivo de modificar uma situação problemática (CONTANDRIOPOULOS ET AL., 2002).

Optou-se pelo desenvolvimento de uma proposta aplicável a municípios com população igual ou superior a 100 mil habitantes, através da seleção de indicadores adequados aos municípios com esse porte. Esta decisão reflete a intenção de investigar casos de maior complexidade na gestão pública, em virtude das singularidades e diversidades dos municípios com tal conformação, e de se estabelecer comparação interna entre os resultados encontrados.

Inicialmente, formou-se um grupo de discussão com integrantes de um programa de pós-graduação em saúde coletiva, de uma instituição federal de ensino superior, composto por pesquisadores, professores e estudantes de diversas áreas da saúde, em nível de mestrado e doutorado. As discussões e reflexões foram realizadas durante seminários de avaliação em saúde e reuniões de um grupo de pesquisa, conformando as etapas iniciais do estudo: elaboração da pergunta de pesquisa, revisão documental e bibliográfica, identificação de objetivos e metas da intervenção, e elaboração da proposta inicial do modelo avaliativo.

Foram realizadas buscas de normativas e publicações científicas relacionadas à Purm. As bases eletrônicas consultadas foram: Saúde Legis, do Ministério da Saúde; PubMed da Medical Literature Analysis and Retrieval System Online (Medline); Literatura Latino-Americana em Ciências da Saúde (Lilacs), via Biblioteca Virtual de Saúde (BVS), Scientific Electronic Library Online (SciELO); e portal de teses e dissertações da Coordenação de Aperfeiçoamento de Pessoal de Nível Superior (Capes). Como termos de busca, foram utilizados: 'Avaliação', 'Gestão em saúde', 'Utilização de medicamentos' e os seus correspondentes em espanhol e inglês.

A revisão conformou o referencial teórico sistematizado e possibilitou a elaboração do modelo teórico para expressar a teoria que orienta a intervenção. O modelo foi debatido e aprovado pelo grupo, e a análise viabilizou: a descrição das responsabilidades da gestão municipal na Purm e dos efeitos esperados da intervenção; a definição da natureza e a magnitude do problema do uso inadequado de medicamentos; a identificação das populações afetadas; e a descrição do contexto.

Esses aspectos orientaram a elaboração do modelo lógico, com a finalidade de explicitar os elementos que constituem e operacionalizam a intervenção, seus objetivos e metas. A sua construção permitiu estabelecer: componentes, subcomponentes, estrutura e processo, além de resultados intermediários e finais da intervenção.

A revisão de literatura também auxiliou na seleção dos componentes da matriz de 
análise, com a inclusão de indicadores utilizados em estudos anteriores. A matriz foi apresentada e discutida com o grupo, que fez sugestões de alteração e reorganização, orientado pela confrontação do objeto avaliado com o previsto, à luz do modelo lógico.

Procedeu-se, então, à validação do modelo teórico, lógico e da matriz de avaliação, por um grupo de especialistas, com a perspectiva de ampliar a análise da plausibilidade e consistência da proposta. Optou-se por utilizar o método de conferência de consenso, que busca conciliar a possibilidade da discussão aberta e, ao mesmo tempo, preservar o anonimato dos participantes (SOUZA; SILVA; HARTZ, 2005).

Foram convidados 13 especialistas provenientes de universidades, do serviço público e da gestão municipal, com formação nas áreas de farmácia, medicina e odontologia. O convite foi orientado pela área de atuação profissional e/ou experiência acadêmica com os temas URM e avaliação em saúde. Dez convidados compuseram o grupo de especialistas e sete colaboraram em todas as etapas da conferência de consenso, mas a participação de todos foi decisiva para a validação do modelo.

A conferência de consenso foi realizada entre os períodos de fevereiro e maio de 2015, em três etapas:

- Pré-análise da proposta: os participantes receberam, via correio eletrônico, a proposta do modelo avaliativo, com as justificativas de cada dimensão, subdimensão e indicadores, para se manifestarem individualmente quanto à 'concordância plena', 'parcial' ou 'não concordância', em cada item. Todos os participantes enviaram sugestões, que foram analisadas e sistematizadas. Os itens com 'concordância parcial' e 'discordância' foram levados para discussão presencial.

- Discussão presencial: foram realizados dois encontros de meio período nas dependências de uma universidade. A síntese da etapa anterior foi apresentada a todos e, em seguida, foi aberto o debate sobre cada item do instrumento de pesquisa. Esses encontros serviram para que os especialistas, pensando em conjunto, propusessem novas adequações ao instrumento.

- Análise final: os comentários e contribuições de comum acordo foram incorporados ao material e enviados aos participantes, que foram convidados a se manifestarem novamente, de forma individual, via correio eletrônico, sobre a 'concordância plena', 'parcial' ou 'não concordância' com a nova proposta. Os participantes concordaram com o resultado final do modelo avaliativo proposto.

A matriz de julgamento foi elaborada de modo a permitir a análise descritiva dos componentes da matriz de análise. Os parâmetros de julgamento estão fundamentados em aspectos normativos, na revisão da literatura e nos acordos firmados entre os especialistas, recebendo as designações 'bom', 'regular' ou 'ruim'. Cada indicador recebeu entre uma e duas medidas, todas com o mesmo grau de importância. Para os indicadores que apresentaram duas medidas binárias, o julgamento 'bom' ou 'ruim' foi atribuído quando houve coincidência na classificação e 'regular' quando discordante. $\mathrm{Na}$ análise dos demais indicadores, assim como das subdimensões, dimensões e da gestão, considerou-se a designação 'bom' quando $50 \%$ dos itens foram classificados como 'bom' e não houve nenhum 'ruim'; e como 'ruim', quando $50 \%$ foram classificados como 'ruim'. Como 'regular', as demais situações.

A pesquisa foi aprovada pelo Comitê de Ética em Pesquisa envolvendo Seres Humanos, do Centro de Hematologia e Hemoterapia do Estado de Santa Catarina (CEP-Hemosc), sob o parecer n ${ }^{\circ} 910.51602$ / dez/2014. 


\section{Resultados e discussão}

As discussões para a definição das atribuições da gestão municipal na Purm se pautaram no conceito de URM e na revisão bibliográfica realizada, considerando o contexto e as possibilidades de atuação da gestão, neste nível. Os documentos normativos que orientaram as discussões foram a PNM, a Política Nacional de Assistência Farmacêutica (PNAF) e o Programa Nacional de Segurança do Paciente (PNSP), que tratam da Purm e da segurança no uso de medicamentos (BRASIL, 1998, 2004, 2013B).

Após intensos debates entre os participantes, concluiu-se que as ações de Purm são intervenções importantes, e devem ser implementadas pela gestão municipal para a prevenção de agravos, proteção à saúde e racionalização de recursos. As discussões levantadas convergem para a compreensão de que as estratégias utilizadas para promover o URM envolvem, entre outras iniciativas, o planejamento e a execução de ações voltadas à organização dos serviços farmacêuticos, a promoção da prescrição racional e o desenvolvimento de um programa educativo voltado para a comunidade (COELHO; PINHEIRO; MAGARINOS-TORRES, 2014).

O modelo teórico apresentado na figura 1 resume a compreensão do URM como um processo que sofre influência das relações da sociedade brasileira com os medicamentos, em especial, nos aspectos socioculturais, de mercado, da formação dos profissionais de saúde e nos aspectos relacionados à gestão.

Figura 1. Modelo teórico das atribuições da gestão municipal, na Purm

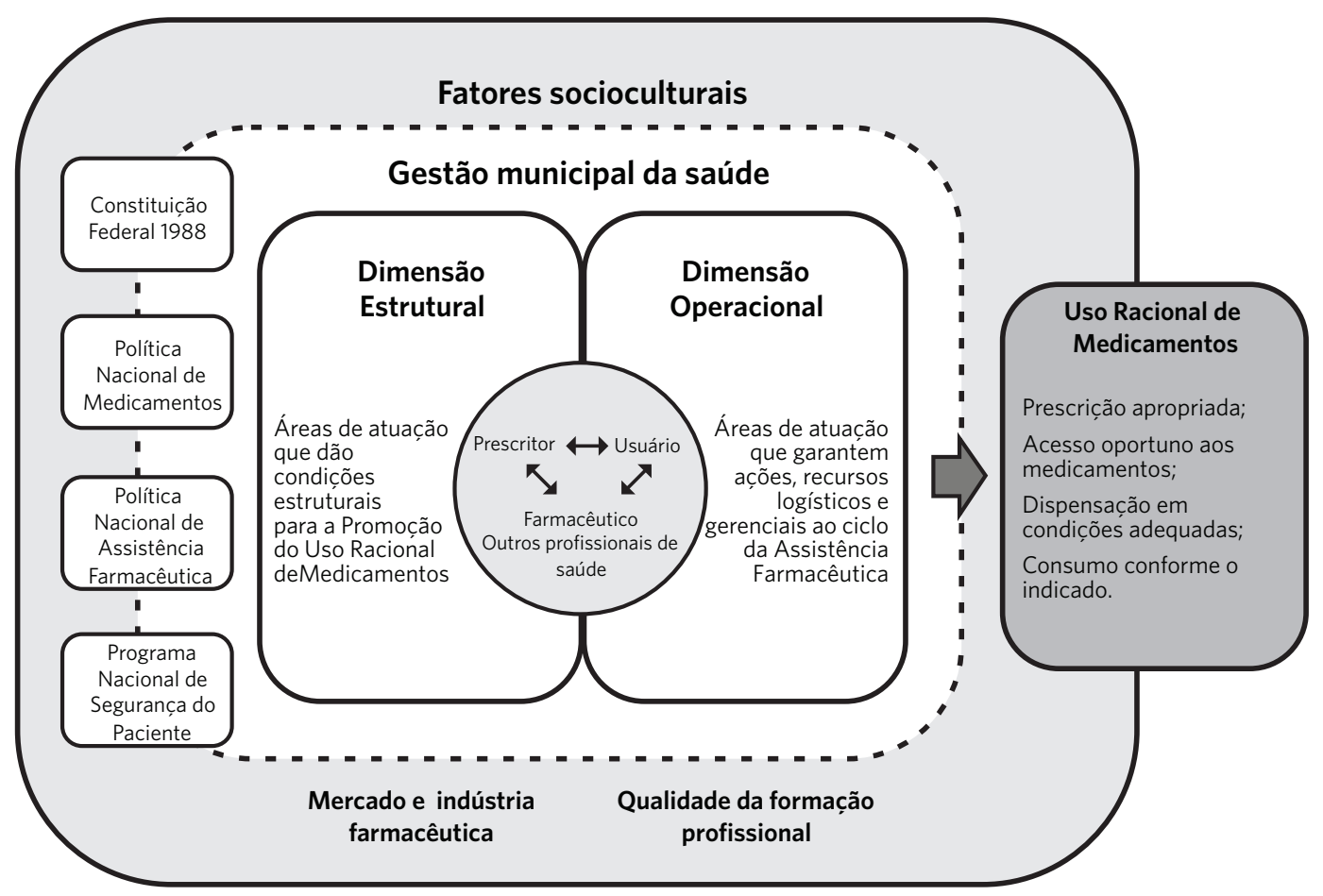

Fonte: Elaboração própria. 
Os fatores socioculturais da população brasileira são determinantes para o padrão de utilização de medicamentos e para a aceitação destes pela sociedade. Os medicamentos têm papel central na abordagem à saúde, vista como algo de valor e objeto desejável, integrado a uma lógica de mercado que estimula o consumo. Este fato é agravado pela atuação do mercado de medicamentos e da indústria farmacêutica, com estratégias de marketing que influenciam as políticas públicas, os profissionais de saúde e os usuários (LEITE; VIEIRA; VEBER, 2008; CAPONI ET AL., 2010).

A formação dos profissionais de saúde é determinante no entendimento do processo saúde-doença e no tipo de assistência que é ofertada à população. $\mathrm{O}$ ensino superior em saúde, centrado no modelo biomédico, dispõe de currículos com uma forte tendência tecnicista, pautada na medicalização, o que compromete a capacidade de julgamento dos profissionais (FERREIRA; FIORINI; CRIVELARO, 2010).

No âmbito da gestão, as determinações legais estabelecidas para o funcionamento do Sistema Único de Saúde (SUS) orientam as decisões político-administrativas e a definição de prioridades no nível municipal. As ações de proteção e promoção da saúde como dever do Estado são discutidas desde a promulgação da Constituição Federal e, a partir de um amplo debate sobre a utilização de medicamentos, foram estabelecidas políticas com diretrizes estratégicas e prioritárias à Purm, com destaque para a PNM e a PNAF. Mais recentemente, o Programa Nacional de Segurança do Paciente (PNSP) estabeleceu ações que visam reduzir os riscos para os pacientes, tais como os decorrentes da utilização de medicamentos (BRASIL, 1988, 1998, 2004, 2013B).

As ações da gestão municipal para a Purm envolvem aspectos estruturais e operacionais. Os estruturais visam dar o suporte necessário à operacionalização das ações de Purm, e referem-se à disponibilidade de recursos humanos, à oferta de estruturas físicas e tecnológicas que auxiliam os profissionais de saúde no cumprimento de suas responsabilidades, além de estarem ligados à instituição de mecanismos de decisão e controle necessários ao planejamento, à coordenação e à execução das ações. Os aspectos operacionais referem-se à capacidade da gestão de ampliar os recursos logísticos e gerenciais necessários à correta execução das etapas do ciclo da Assistência Farmacêutica (AF), para assegurar o acesso e o uso de medicamentos seguros, eficazes e de qualidade (BARRETO; GUIMARÃES, 2010).

As ações da gestão devem ser voltadas para os atores responsáveis pela operacionalização do URM: os prescritores, os demais profissionais de saúde e a população. A prescrição é a primeira etapa da utilização de medicamentos, e a relação entre prescritor e usuário tem papel crucial para a Purm. Para ser efetiva, a prescrição deve ser vista de maneira satisfatória por ambos, e a adesão à terapia é dependente das trocas de informações, das vivências e da adequada orientação no momento da consulta (PEPE; OSÓRIO-DE-CASTRO, 2000).

A Purm é uma atividade multidisciplinar, e todos os profissionais de saúde devem contribuir com esse fim. Em especial, deve-se evidenciar a relação do usuário com o farmacêutico, que se encontra na interface da dispensação e da utilização dos medicamentos, fazendo a conferência da prescrição, orientando o usuário e monitorando os resultados da terapia medicamentosa. Merece destaque, também, a relação entre o prescritor e os demais profissionais de saúde, para que, através da combinação de conhecimentos e da complementaridade de saberes, sejam alcançados resultados mais eficientes na utilização de medicamentos (PEPE; OSÓRIO-DE-CASTRO, 2000; MARIN ET AL., 2003).

Como resultado das ações da gestão municipal, espera-se contribuir para o URM através: da prescrição de medicamentos apropriada, do acesso oportuno da população aos medicamentos, da dispensação em 
condições adequadas; e consumo dos medicamentos, pela comunidade, conforme o indicado no serviço de saúde.

A identificação e o detalhamento dos componentes, assim como as atividades e os resultados esperados mediante a intervenção, estão expressos no modelo lógico (figura 2). Os elementos foram acordados com o objetivo de identificar as estruturas necessárias e as atividades com execuções previstas. Concluiu-se que, para promover o URM, o gestor municipal, nas suas atribuições, deve:

- Realizar ações centradas no provimento de informação sobre a utilização adequada de medicamentos, voltadas aos profissionais de saúde e à comunidade, na tentativa de minimizar possíveis deficiências na formação profissional, capacitar os recursos humanos para o cumprimento das suas responsabilidades e assegurar que a população tenha acesso a informações sobre a utilização de medicamentos (MARIN ET AL., 2003; EV; GONÇALVES, 2014);

- Garantir a disponibilização de recursos humanos e meios físicos necessários ao atendimento da população; contribuir com o URM e colaborar com a segurança na utilização de medicamentos nos serviços de saúde, assim como prevenir a automedicação e os problemas com uso inadequado de medicamentos;

- Instituir mecanismos de decisão e controle com o intuito de auxiliar no planejamento, na organização e na execução de ações de Purm, além de orientar a tomada de decisão nos serviços de saúde (Coelho; PINHEIRO; MAGARINOS-TORRES, 2014);

- Garantir o cumprimento das etapas de seleção, programação, aquisição, armazenamento, distribuição, prescrição e dispensação de medicamentos, as quais visam garantir o acesso oportuno da população aos medicamentos e promover seu uso racional (MARIN ET AL., 2003).

A análise do conceito de URM e das responsabilidades no nível municipal permitiu compreender a importância da atuação da gestão na Purm. Ao ofertar o suporte necessário à realização das ações, e sua execução de forma coordenada e simultânea, em diversos pontos, no processo de utilização de medicamentos, amplia-se o escopo de atuação e o potencial de sucesso da Purm, uma vez que não existe uma ferramenta única para a resolução dos problemas relacionados à má utilização de medicamentos. 
Figura 2. Modelo lógico das atribuições da gestão municipal, na Purm

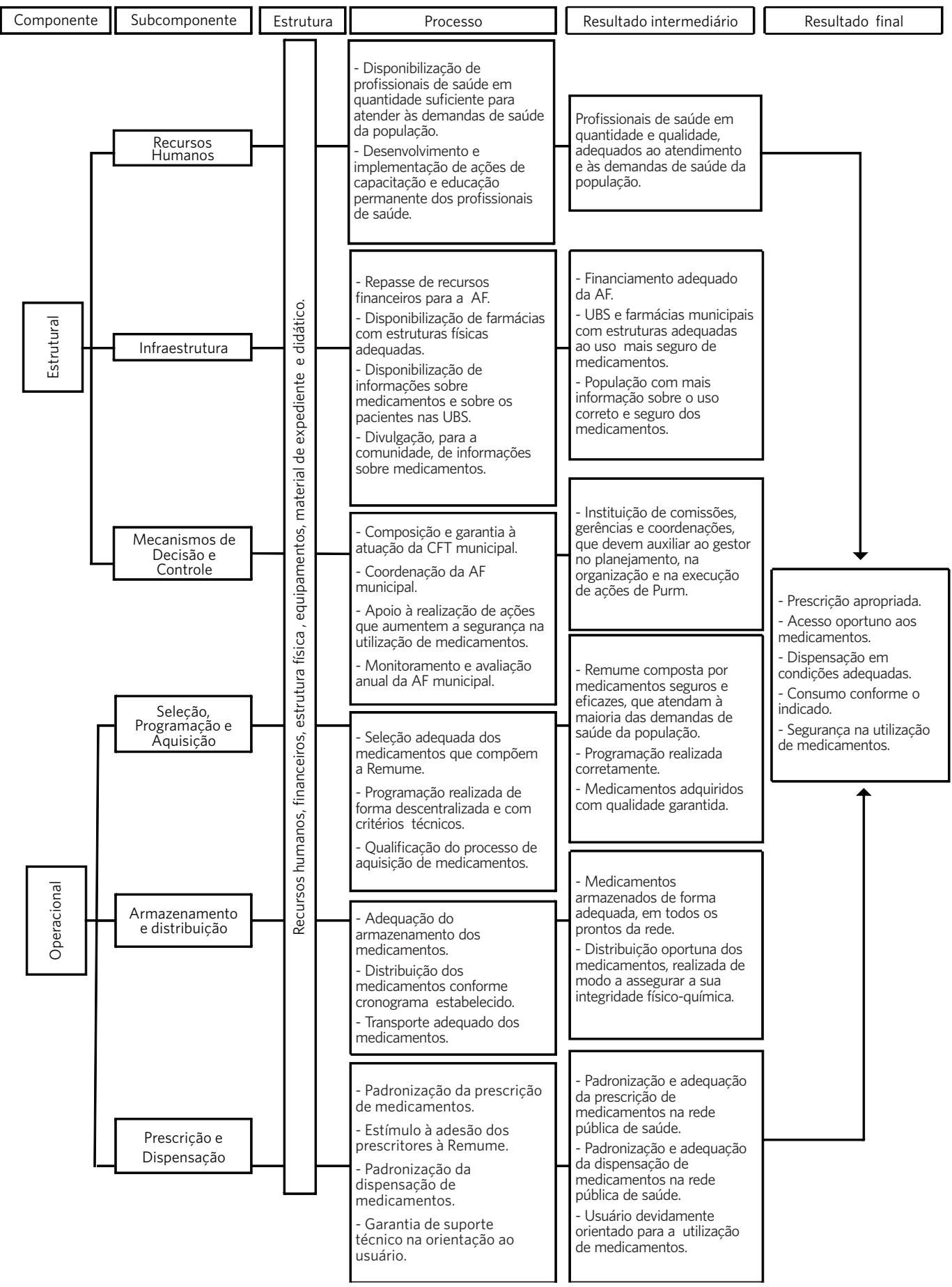

Fonte: Elaboração própria.

AF - Assistência Farmacêutica

CFT - Comissão de Farmácia e Terapêutica

Remume - Relação Municipal de Medicamentos Essenciais

Purm - Promocão do Uso Racional de Medicamentos

UBS - Unidades Básicas de Saúde. 


\section{A matriz de avaliação final foi composta listados no quadro 1, com suas respectivas pelas dimensões Estrutural e Operacional, justificativas, medidas e parâmetros.}

com 6 subdimensões e 28 indicadores

Quadro 1. Matriz de análise das ações da gestão municipal na Purm. Brasil, 2015

\begin{tabular}{|c|c|c|c|c|c|c|}
\hline & & Indicador & Justificativa & Medida & Parâmetro & Fonte \\
\hline \multirow{4}{*}{ 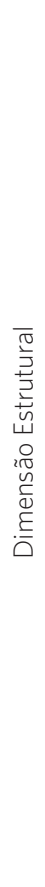 } & \multirow{4}{*}{ 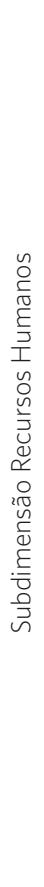 } & $\begin{array}{l}\text { Cobertura dos } \\
\text { profissionais da } \\
\text { atenção básica. }\end{array}$ & $\begin{array}{l}\text { A manutenção da relação adequa- } \\
\text { da entre o número de profissionais } \\
\text { da atenção básica e a população } \\
\text { do município contribui com o } \\
\text { URM (NUNES; AMADOR; HEINECK, } \\
\text { 2008). }\end{array}$ & $\begin{array}{l}\text { Relação entre o } n^{\circ} \text { de } \\
\text { equipes de saúde da } \\
\text { família, ou equivalentes, e } \\
\text { a população residente. }\end{array}$ & $\begin{array}{l}\text { Bom (5): } \leq 3000 \\
\text { Regular (3): 3001-4000; } \\
\text { Ruim (0): >4000. }\end{array}$ & $\begin{array}{l}\text { Entrevis- } \\
\text { ta/IBGE. }\end{array}$ \\
\hline & & $\begin{array}{l}\text { Cobertura de } \\
\text { farmacêuticos } \\
\text { atuando na } \\
\text { atenção básica. }\end{array}$ & $\begin{array}{l}\text { Pressupõe o direito de acesso da } \\
\text { população aos serviços farmacêu- } \\
\text { ticos (CFF, 2010). }\end{array}$ & $\begin{array}{l}\text { Relação entre o } n^{\circ} \text { de } \\
\text { farmacêuticos atuando na } \\
\text { atenção básica e a popu- } \\
\text { lação residente. }\end{array}$ & $\begin{array}{l}\operatorname{Bom}(5): \leq 10.000 \\
\operatorname{Ruim}(0):>10.000\end{array}$ & $\begin{array}{l}\text { Entrevis- } \\
\text { ta/IBGE. }\end{array}$ \\
\hline & & $\begin{array}{l}\text { Qualificação } \\
\text { dos profissio- } \\
\text { nais de saúde } \\
\text { no ingresso ao } \\
\text { serviço público, } \\
\text { sobre o URM. }\end{array}$ & $\begin{array}{l}\text { A gestão deve desenvolver ações } \\
\text { a fim de minimizar os efeitos de } \\
\text { possíveis deficiências da formação } \\
\text { profissional (IVAMA-BRUMMELL; } \\
\text { LYRA JÚNIOR; SAKAI, 2014). }\end{array}$ & $\begin{array}{l}\text { Realização de capaci- } \\
\text { tações sobre o URM no } \\
\text { prazo de até seis meses } \\
\text { após o ingresso. }\end{array}$ & $\begin{array}{l}\text { Bom (5): Sim; } \\
\text { Ruim (0): Não. }\end{array}$ & $\begin{array}{l}\text { Lista de } \\
\text { presença } \\
\text { das capa- } \\
\text { citações. }\end{array}$ \\
\hline & & $\begin{array}{l}\text { Educação } \\
\text { permanente } \\
\text { oferecida aos } \\
\text { profissionais de } \\
\text { saúde, sobre o } \\
\text { URM. }\end{array}$ & $\begin{array}{l}\text { O gestor municipal deve manter } \\
\text { um conjunto de trabalhadores } \\
\text { atentos e capacitados para a Purm } \\
\text { (BRASIL, 1998). }\end{array}$ & $\begin{array}{l}\text { Realização de educação } \\
\text { permanente dos profis- } \\
\text { sionais de saúde sobre o } \\
\text { URM, nos últimos dois } \\
\text { anos. }\end{array}$ & $\begin{array}{l}\operatorname{Bom}(5): \text { Sim; } \\
\text { Ruim (0): Não. }\end{array}$ & $\begin{array}{l}\text { Registro } \\
\text { das capa- } \\
\text { citações. }\end{array}$ \\
\hline \multirow{2}{*}{ 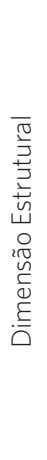 } & \multirow{2}{*}{ 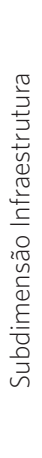 } & $\begin{array}{l}\text { Financiamento } \\
\text { da AF. }\end{array}$ & $\begin{array}{l}\text { A gestão municipal deve financiar } \\
\text { adequadamente a compra dos } \\
\text { medicamentos, proporcionando } \\
\text { à população o acesso aos medi- } \\
\text { camentos e contribuindo, dessa } \\
\text { forma, com o URM. }\end{array}$ & $\begin{array}{l}\text { Valor investido na com- } \\
\text { pra de medicamentos do } \\
\text { componente básico da } \\
\text { AF, no último ano. }\end{array}$ & $\begin{array}{l}\text { Bom (5): Investiu, no mínimo, o } \\
\text { valor normatizado; } \\
\text { Ruim (0): Investiu menos que o } \\
\text { valor normatizado. }\end{array}$ & $\begin{array}{l}\text { Relatório } \\
\text { anual de } \\
\text { gestão. }\end{array}$ \\
\hline & & $\begin{array}{l}\text { Ambiente } \\
\text { destinado aos } \\
\text { serviços farma- } \\
\text { cêuticos. }\end{array}$ & $\begin{array}{l}\text { A disponibilização de farmácias } \\
\text { em boas condições contribui para } \\
\text { a adequada execução dos serviços } \\
\text { farmacêuticos. }\end{array}$ & $\begin{array}{l}\text { Porcentagem de farmá- } \\
\text { cias que atendem às es- } \\
\text { pecificações de estrutura } \\
\text { definidas pela Resolução } \\
\text { de Diretoria Colegiada } \\
\text { (RCD) no 44/2009. }\end{array}$ & $\begin{array}{l}\text { Bom (5): 100\%; } \\
\text { Regular (3): } 99 \%-70 \% \\
\text { Ruim (0): }<70 \%\end{array}$ & $\begin{array}{l}\text { Visitas } \\
\text { técnicas. }\end{array}$ \\
\hline
\end{tabular}


Quadro 1. (cont.)

\begin{tabular}{|c|c|c|c|c|c|c|c|}
\hline \multirow{3}{*}{ 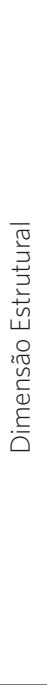 } & \multirow{3}{*}{ 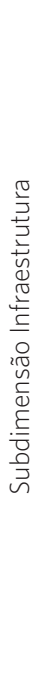 } & \multirow{3}{*}{$\begin{array}{l}\text { Suporte à deci- } \\
\text { são clínica. } \\
\\
\text { Estratégias de } \\
\text { divulgação de } \\
\text { informações } \\
\text { sobre medi- } \\
\text { camentos à } \\
\text { comunidade. }\end{array}$} & \multirow{3}{*}{$\begin{array}{l}\text { A gestão municipal deve disponi- } \\
\text { bilizar aos profissionais de saúde } \\
\text { os recursos necessários para dar } \\
\text { suporte à decisão clínica, com o } \\
\text { intuito de contribuir com o URM. } \\
\text { A população também deve ser } \\
\text { alvo das ações para a Purm, já que } \\
\text { muitas vezes não tem informações } \\
\text { sobre os riscos e os benefícios do } \\
\text { uso dos medicamentos (WHO, } \\
2002 ; \text { MARIN ET AL., 2003). }\end{array}$} & \multirow{2}{*}{$\begin{array}{l}\text { Porcentagem de Unida- } \\
\text { des Básicas de Saúde } \\
\text { (UBS) que contam com } \\
\text { cópia do FT ou sistemas } \\
\text { online de informações de } \\
\text { medicamentos. } \\
\text { Porcentagem de UBS que } \\
\text { utilizam um sistema de } \\
\text { prontuários eletrônicos. }\end{array}$} & $\begin{array}{l}\text { Bom (5): 100\%; } \\
\text { Regular (3): } \\
\text { 99\%-50\%; } \\
\text { Ruim (0):<50\%. }\end{array}$ & $\begin{array}{l}\sum \text { dos esco- } \\
\text { res: } \\
\text { 10-8: Bom } \\
\text { (5); } \\
\text { 6: Regular (3); } \\
\text { 3-0: Ruim (0). }\end{array}$ & $\begin{array}{l}\text { Visitas } \\
\text { técnicas. }\end{array}$ \\
\hline & & & & & \multicolumn{2}{|l|}{$\begin{array}{l}\text { Bom (5): } \geq 80 \% \\
\text { Regular (3): } \\
79 \%-50 \% \\
\text { Ruim (0): } \\
<50 \%\end{array}$} & $\begin{array}{l}\text { Visitas } \\
\text { técnicas. }\end{array}$ \\
\hline & & & & $\begin{array}{l}\text { - Divulgação de informa- } \\
\text { ções na mídia; } \\
\text { - Disponibilização de } \\
\text { materiais de apoio aos } \\
\text { profissionais de saúde. }\end{array}$ & \multicolumn{2}{|c|}{$\begin{array}{l}\text { Bom (5): Sim para as duas ques- } \\
\text { tões; } \\
\text { Regular (3): Sim para uma das } \\
\text { questões; } \\
\text { Ruim (0): Não realiza. }\end{array}$} & $\begin{array}{l}\text { Materiais } \\
\text { de divul- } \\
\text { gação/en- } \\
\text { trevista. }\end{array}$ \\
\hline \multirow{6}{*}{ 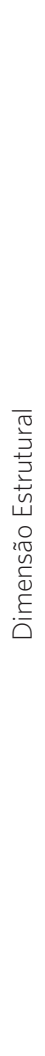 } & \multirow{6}{*}{ 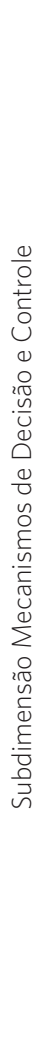 } & $\begin{array}{l}\text { Existência e } \\
\text { composição da } \\
\text { CFT. }\end{array}$ & $\begin{array}{l}\text { Expressa o interesse da gestão em } \\
\text { selecionar medicamentos seguros, } \\
\text { eficazes e adequados às neces- } \\
\text { sidades da população (MARIN ET } \\
\text { AL., 2003). }\end{array}$ & $\begin{array}{l}\text { - CFT formada minima- } \\
\text { mente por médico, enfer- } \\
\text { meiro e farmacêutico; } \\
\text { - CFT formalizada na } \\
\text { secretaria. }\end{array}$ & \multicolumn{2}{|c|}{$\begin{array}{l}\text { Bom (5): Sim para as duas questões; } \\
\text { Regular (3): Sim para a primeira } \\
\text { questão e Não para a segunda; } \\
\text { Ruim (0): Não para a primeira } \\
\text { questão. }\end{array}$} & $\begin{array}{l}\text { Portaria } \\
\text { de insti- } \\
\text { tuição/ } \\
\text { atas das } \\
\text { reuniões. }\end{array}$ \\
\hline & & $\begin{array}{l}\text { Regularidade } \\
\text { de atuação da } \\
\text { CFT. }\end{array}$ & $\begin{array}{l}\text { Demonstra o grau de interesse } \\
\text { na política de uso racional e o } \\
\text { compromisso dos seus membros } \\
\text { com as atividades (MENDES ET AL., } \\
\text { 2015). }\end{array}$ & $\begin{array}{l}\text { Número de reuniões reali- } \\
\text { zadas no último ano. }\end{array}$ & \multicolumn{2}{|l|}{$\begin{array}{l}\operatorname{Bom}(5): \geq 6 \\
\operatorname{Ruim}(0):<6\end{array}$} & $\begin{array}{l}\text { Atas das } \\
\text { reuniões. }\end{array}$ \\
\hline & & $\begin{array}{l}\text { Coordenação } \\
\text { da AF. }\end{array}$ & $\begin{array}{l}\text { Pressupõe a preocupação da } \\
\text { gestão municipal na estruturação } \\
\text { e na qualificação dos serviços } \\
\text { farmacêuticos. }\end{array}$ & $\begin{array}{l}\text { Existência da coordena- } \\
\text { ção da AF na Secretaria } \\
\text { Municipal de Saúde. }\end{array}$ & \multicolumn{2}{|l|}{$\begin{array}{l}\text { Bom (5): Sim; } \\
\text { Ruim (0): Não. }\end{array}$} & $\begin{array}{l}\text { Entrevis- } \\
\text { tas. }\end{array}$ \\
\hline & & $\begin{array}{l}\text { Estratégias para } \\
\text { o gerenciamen- } \\
\text { to da segurança } \\
\text { dos pacientes }\end{array}$ & $\begin{array}{l}\text { Dispõem sobre a necessidade de } \\
\text { instituir meios de gerenciamento } \\
\text { de riscos na utilização de medi- } \\
\text { camentos (MARIN ET AL., 2003; }\end{array}$ & $\begin{array}{l}\text { Existência de um Núcleo } \\
\text { de Segurança do Paciente } \\
\text { (NSP) no município. }\end{array}$ & $\begin{array}{l}\text { Bom (5): Sim; } \\
\text { Ruim (0): Não. }\end{array}$ & $\begin{array}{l}\sum \text { dos esco- } \\
\text { res: } \\
\text { 10: Bom (5); } \\
\text { 5: Regular (3); }\end{array}$ & Entrevista. \\
\hline & & $\begin{array}{l}\text { nos serviços de } \\
\text { saúde. }\end{array}$ & BRASIL, 2013A). & $\begin{array}{l}\text { Definição do fluxo de } \\
\text { farmacovigilância. }\end{array}$ & $\begin{array}{l}\text { Bom (5): Sim; } \\
\text { Ruim (0): Não. }\end{array}$ & $0: \operatorname{Ruim}(0)$ & Entrevista. \\
\hline & & $\begin{array}{l}\text { Monitoramento } \\
\text { e avaliação da } \\
\text { AF. }\end{array}$ & $\begin{array}{l}\text { O monitoramento e a avaliação da } \\
\text { AF são indispensáveis à garantia } \\
\text { da qualidade dos serviços oferta- } \\
\text { dos (WHO, 2002). }\end{array}$ & $\begin{array}{l}\text { - Aplicação anual de um } \\
\text { instrumento de avaliação } \\
\text { da AF; } \\
\text { - Devolução dos resulta- } \\
\text { dos aos profissionais de } \\
\text { saúde. }\end{array}$ & \multicolumn{2}{|c|}{$\begin{array}{l}\text { Bom (5): Sim para as duas ques- } \\
\text { tões; } \\
\text { Regular (3): Sim para a primeira } \\
\text { questão e não para a segunda; } \\
\text { Ruim (0): Não faz. }\end{array}$} & $\begin{array}{l}\text { Relatório } \\
\text { da última } \\
\text { avaliação/ } \\
\text { entrevista. }\end{array}$ \\
\hline
\end{tabular}


Quadro 1. (cont.)

\begin{tabular}{|c|c|c|c|c|c|c|}
\hline \multirow{6}{*}{ 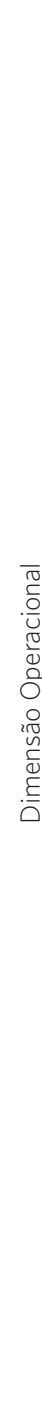 } & \multirow{6}{*}{ 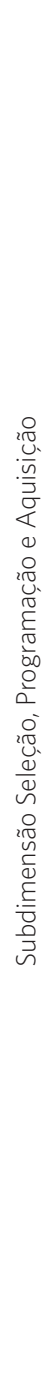 } & $\begin{array}{l}\text { Adequação da } \\
\text { Remume. }\end{array}$ & $\begin{array}{l}\text { Expressa a adequação do processo } \\
\text { de seleção de medicamentos, com } \\
\text { base na Relação Nacional de Medica- } \\
\text { mentos Essenciais (Rename) (BRASIL, } \\
\text { 1998). }\end{array}$ & $\begin{array}{l}\text { Porcentagem de medi- } \\
\text { camentos constantes na } \\
\text { Remume e que também } \\
\text { constam na Rename }\end{array}$ & $\begin{array}{l}\text { Bom (5): } \geq 80 \% \\
\text { Regular (3): } 79 \%-60 \% \\
\text { Ruim (0): }<60 \%\end{array}$ & $\begin{array}{l}\text { Rename/ } \\
\text { Remume. }\end{array}$ \\
\hline & & $\begin{array}{l}\text { Programação de } \\
\text { medicamentos. }\end{array}$ & $\begin{array}{l}\text { A gestão municipal deve garantir } \\
\text { que a programação de medicamen- } \\
\text { tos seja realizada adequadamente, } \\
\text { contribuindo para o acesso da } \\
\text { população aos medicamentos e, } \\
\text { consequentemente, para a PURM } \\
\text { (BRASIL, 2006). }\end{array}$ & $\begin{array}{l}\text { Programação de medica- } \\
\text { mentos realizada: } \\
\text { - UBS; } \\
\text { - Nível Central. } \\
\text { Programação de medica- } \\
\text { mentos nas UBS realizada } \\
\text { com critérios técnicos. }\end{array}$ & $\begin{array}{ll}\text { Bom (5): Sim para } & \sum \text { dos escores: } \\
\text { as duas questões; } & \text { 10: Bom (5); } \\
\text { Ruim (0): Outras } & \text { 5: Regular (3); } \\
\text { possibilidades. } & \text { 0: Ruim (0); } \\
& \\
\text { Bom (5): Sim; } & \\
\text { Ruim (0): Não. } & \end{array}$ & Entrevistas. \\
\hline & & $\begin{array}{l}\text { Cumprimento da } \\
\text { programação na } \\
\text { compra de medi- } \\
\text { camentos. }\end{array}$ & $\begin{array}{l}\text { A aquisição de medicamentos deve } \\
\text { ser sempre orientada pela progra- } \\
\text { mação, e cortes realizados podem } \\
\text { comprometer a disponibilidade de } \\
\text { medicamentos (BRASIL, 2006). }\end{array}$ & $\begin{array}{l}\text { Porcentagem de itens } \\
\text { adquiridos conforme a } \\
\text { programação, na última } \\
\text { licitação municipal. }\end{array}$ & $\begin{array}{l}\text { Bom (5): Sim; } \\
\text { Não (0): Qualquer outra possibi- } \\
\text { lidade. }\end{array}$ & $\begin{array}{l}\text { Programa- } \\
\text { ção/edital } \\
\text { de licitação. }\end{array}$ \\
\hline & & $\begin{array}{l}\text { Padronização de } \\
\text { compras. }\end{array}$ & $\begin{array}{l}\text { Eventuais aquisições de itens que } \\
\text { se encontram fora das listas de } \\
\text { padronização devem ser efetuadas } \\
\text { somente com justificativas clínicas } \\
\text { específicas (MARIN ET AL., 2003). }\end{array}$ & $\begin{array}{l}\text { Compra de medicamen- } \\
\text { tos que não pertencem à } \\
\text { Remume, com pareceres } \\
\text { técnicos da CFT. }\end{array}$ & $\begin{array}{l}\text { Bom (5): Sim; } \\
\operatorname{Ruim}(0): \text { Não. }\end{array}$ & $\begin{array}{l}\text { Pareceres } \\
\text { da CFT. }\end{array}$ \\
\hline & & $\begin{array}{l}\text { Qualificação dos } \\
\text { fornecedores. }\end{array}$ & $\begin{array}{l}\text { O município deve fazer avaliações } \\
\text { de desempenho dos seus fornece- } \\
\text { dores, a fim de garantir a qualidade } \\
\text { e o abastecimento dos medicamen- } \\
\text { tos (MARIN ET AL., 2003). }\end{array}$ & $\begin{array}{l}\text { - Elaboração de um registro } \\
\text { do recebimento dos medi- } \\
\text { camentos; } \\
\text { - Análise do desempenho } \\
\text { dos fornecedores. }\end{array}$ & $\begin{array}{l}\text { Bom (5): Sim para as duas ques- } \\
\text { tões; } \\
\text { Regular (3): Sim para a primeira } \\
\text { questão e Não para a segunda; } \\
\text { Ruim (0): Não faz. }\end{array}$ & $\begin{array}{l}\text { Registro } \\
\text { elaborado/ } \\
\text { entrevistas. }\end{array}$ \\
\hline & & $\begin{array}{l}\text { Qualidade do } \\
\text { processo de } \\
\text { licitação. }\end{array}$ & $\begin{array}{l}\text { Expressa a necessidade da exigên- } \\
\text { cia da documentação sanitária reco- } \\
\text { mendada pelo Ministério da Saúde } \\
\text { nos editais de compra, visando } \\
\text { garantir a procedência e a qualidade } \\
\text { dos medicamentos (BRASIL, 2006). }\end{array}$ & $\begin{array}{l}\text { Edital de licitação com os } \\
\text { requisitos que assegurem } \\
\text { a qualidade dos medica- } \\
\text { mentos. }\end{array}$ & $\begin{array}{l}\text { Bom (5): Sim; } \\
\operatorname{Ruim}(0): \text { Não. }\end{array}$ & $\begin{array}{l}\text { Edital de } \\
\text { licitação. }\end{array}$ \\
\hline \multirow{3}{*}{ 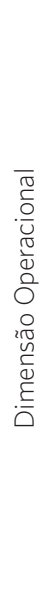 } & \multirow{3}{*}{ 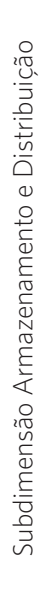 } & $\begin{array}{l}\text { Adequação do } \\
\text { armazenamento. }\end{array}$ & $\begin{array}{l}\text { A gestão municipal deve garantir que } \\
\text { todos os pontos de armazenamento } \\
\text { de medicamentos possuam ambien- } \\
\text { tes adequados, capazes de garantir } \\
\text { sua integridade física e química (MEN- } \\
\text { DES, 2013). }\end{array}$ & $\begin{array}{l}\text { Porcentagem de farmácias } \\
\text { municipais com os ins- } \\
\text { trumentos mínimos para } \\
\text { garantir a integridade física } \\
\text { dos medicamentos. }\end{array}$ & $\begin{array}{l}\text { Bom (5): 100\%; } \\
\text { Regular (3): 99\%-80\%; } \\
\text { Ruim (0): <80\% }\end{array}$ & $\begin{array}{l}\text { Visitas } \\
\text { técnicas. }\end{array}$ \\
\hline & & $\begin{array}{l}\text { Controle de } \\
\text { estoque. }\end{array}$ & $\begin{array}{l}\text { Dispõe a respeito da necessidade } \\
\text { de manter informações confiáveis } \\
\text { sobre a movimentação física e } \\
\text { financeira de estoques de medica- } \\
\text { mentos (MARIN ET AL., 2003). }\end{array}$ & $\begin{array}{l}\text { Porcentagem dos erros } \\
\text { encontrados no relatório } \\
\text { de inventário realizado no } \\
\text { último ano. }\end{array}$ & $\begin{array}{l}\text { Bom ( } 5): \leq 10 \% \text {; } \\
\text { Ruim (0): }>10 \% \text { ou não fez inventá- } \\
\text { rio no último ano. }\end{array}$ & $\begin{array}{l}\text { Último } \\
\text { relatório de } \\
\text { inventário. }\end{array}$ \\
\hline & & $\begin{array}{l}\text { Descarte ade- } \\
\text { quado de medi- } \\
\text { camentos. }\end{array}$ & $\begin{array}{l}\text { O descarte adequado de medi- } \\
\text { camentos evita a contaminação } \\
\text { ambiental, que pode causar eventos } \\
\text { adversos à saúde. }\end{array}$ & $\begin{array}{l}\text { Contrato vigente com } \\
\text { empresa que deve fazer o } \\
\text { descarte. }\end{array}$ & $\begin{array}{l}\text { Bom (5): Sim; } \\
\operatorname{Ruim}(0) \text { : Não. }\end{array}$ & $\begin{array}{l}\text { Contrato } \\
\text { vigente. }\end{array}$ \\
\hline
\end{tabular}


Quadro 1. (cont.)

\begin{tabular}{|c|c|c|c|c|c|c|c|}
\hline 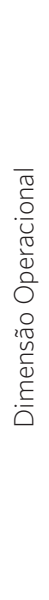 & 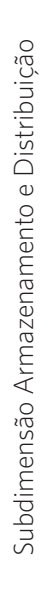 & $\begin{array}{l}\text { Adequação do } \\
\text { transporte de } \\
\text { medicamentos. }\end{array}$ & $\begin{array}{l}\text { Demonstra o interesse da gestão } \\
\text { em fazer o planejamento e ga- } \\
\text { rantir o abastecimento adequado } \\
\text { de medicamentos nas farmácias } \\
\text { municipais (CFF, 2010). } \\
\text { As atividades de transporte devem } \\
\text { garantir que os medicamentos } \\
\text { sejam entregues a unidades de } \\
\text { saúde, obedecendo aos critérios } \\
\text { adequados, de modo a não com- } \\
\text { prometer a sua qualidade (MARIN } \\
\text { et al., 2003). }\end{array}$ & $\begin{array}{l}\text { Cronograma estabeleci- } \\
\text { do, de abastecimento de } \\
\text { medicamentos. } \\
\text { Porcentagem de entregas } \\
\text { realizadas com mais de } \\
15 \text { dias de atrasos, nos } \\
\text { últimos } 6 \text { meses. } \\
\text { Disposição de veículo } \\
\text { adequado, que atenda às } \\
\text { boas práticas de trans- } \\
\text { porte. }\end{array}$ & $\begin{array}{l}\text { Bom (5): Sim; } \\
\text { Ruim (0): Não. } \\
\text { Bom (5): } \leq 10 \% \text {; } \\
\text { Regular (3): } \\
\text { 11\%-20\%; } \\
\text { Ruim (0): >20\% } \\
\text { ou não registra. } \\
\text { Bom (5): Sim; } \\
\text { Ruim (0): Não. }\end{array}$ & $\begin{array}{l}\sum \text { dos esco- } \\
\text { res: } \\
\text { 10: Bom (5); } \\
\text { 5: Regular (3); } \\
\text { 0: Ruim (0). }\end{array}$ & $\begin{array}{l}\text { Registro } \\
\text { das entre- } \\
\text { gas nos } \\
\text { últimos } 15 \\
\text { dias. } \\
\text { Visita } \\
\text { técnica. }\end{array}$ \\
\hline 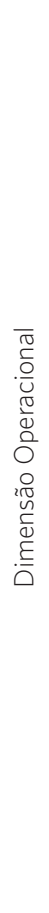 & 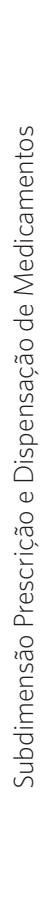 & $\begin{array}{l}\text { Padronização } \\
\text { da prescrição } \\
\text { de medicamen- } \\
\text { tos. }\end{array}$ & $\begin{array}{l}\text { Dispõe sobre a elaboração de } \\
\text { normativas ou procedimentos que } \\
\text { visam orientar, disciplinar e padro- } \\
\text { nizar a prescrição de medicamen- } \\
\text { tos (BRASIL, 2004; CFF, 2010). } \\
\text { A disseminação do uso das listas } \\
\text { de medicamentos essenciais } \\
\text { permite a melhoria do acesso, } \\
\text { da qualidade e da efetividade na } \\
\text { utilização de medicamentos (EV; } \\
\text { GUIMARÃES, 2014). } \\
\text { Devem ser elaborados e divulga- } \\
\text { dos manuais e portarias que nor- } \\
\text { matizem a dispensação, segundo } \\
\text { a legislação sanitária vigente (CFF, } \\
\text { 2010). } \\
\text { A orientação é fundamental na } \\
\text { adesão ao tratamento e ao URM. } \\
\text { Quando os usuários não são ade- } \\
\text { quadamente esclarecidos sobre o } \\
\text { seu regime terapêutico, os riscos } \\
\text { de erros na medicação aumentam } \\
\text { muito (EV; GUIMARÃES, } 2014 \text { ). }\end{array}$ & $\begin{array}{l}\text { Disponibilização de far- } \\
\text { macêuticos com atribui- } \\
\text { ção de divulgar e incenti- } \\
\text { var a adesão à Remume. }\end{array}$ & $\begin{array}{l}\text { Bom: }=100 \% \text {; } \\
\text { Ruim: Qualquer o } \\
\text { dade. }\end{array}$ & ra possibili- & $\begin{array}{l}\text { Norma, } \\
\text { manuais } \\
\text { ou proce- } \\
\text { dimento. }\end{array}$ \\
\hline
\end{tabular}

Fonte: Elaboração própria.

AF - Assistência Farmacêutica

CFF - Conselho Federal de Farmácia

CFT - Comissão de Farmácia e Terapêutica

FT - Formulário Terapêutico

NSP - Núcleo de Segurança do Paciente

Purm - Promoção do Uso Racional de Medicamentos

Remume - Relação Municipal de Medicamentos Essenciais

UBS - Unidades Básicas de Saúde

URM - Uso Racional de Medicamentos. 
A dimensão Estrutural contempla a análise dos aspectos gerenciais básicos à organização de ações de Purm: Recursos Humanos; Infraestrutura; Mecanismos de Decisão e Controle.

A escassez de profissionais de saúde e as falhas na sua qualificação podem favorecer o processo de automedicação e o uso inadequado de medicamentos. Nesta perspectiva, a subdimensão 'Recursos Humanos' propõe a análise da quantidade de profissionais de saúde disponíveis para atender às demandas de saúde da população, bem como do esforço da gestão para qualificá-los adequadamente quanto ao cumprimento das suas responsabilidades na Purm.

Na subdimensão 'Infraestrutura', propõe-se a análise dos investimentos, dos aspectos do ambiente de trabalho, do aporte tecnológico e dos materiais necessários para o bom funcionamento das equipes de saúde e para a Purm.

A subdimensão 'Mecanismos de Decisão e Controle' está relacionada à complexidade e à diversidade das atividades relacionadas à Purm, que requerem a criação de meios para auxiliar o gestor no planejamento, no desenvolvimento e no acompanhamento das atividades (COELHO; PINHEIRO; MAGARINOS-TORRES, 2014). A subdimensão foi composta por cinco indicadores, relacionados a existência e atuação da Comissão de Farmácia e Terapêutica (CFT) do município, da coordenação da AF, gerenciamento da segurança dos pacientes nos serviços de saúde e monitoramento e avaliação da AF.

A dimensão Operacional visa identifica a atuação da gestão municipal em todo o ciclo da assistência farmacêutica. A subdimensão 'Seleção, Programação e Aquisição' analisa a capacidade da gestão para selecionar e adquirir adequadamente os medicamentos utilizados no município e que serão distribuídos à população, garantido, assim, a disponibilidade adequada de medicamentos mais seguros e de qualidade, que vão contribuir para a Purm no município.

A subdimensão 'Armazenamento e Distribuição' analisa a capacidade da gestão para assegurar a qualidade e a disponibilidade oportuna e regular dos medicamentos. Ela foi composta por indicadores relacionados à adequação do armazenamento nas farmácias municipais, ao controle de estoque, ao descarte e à regularidade de distribuição e qualidade do transporte.

A subdimensão 'Prescrição e Dispensação de medicamentos' dispõe que o gestor deve instituir meios de padronização e melhorias nos serviços de prescrição de medicamentos, dispensação e orientação ao usuário, através da padronização das atividades, do estímulo à utilização da Relação Municipal de Medicamentos Essenciais (Remume) e da disponibilização de suporte ao usuário.

A operacionalização das etapas do ciclo da AF foi considerada como requisito básico para a garantia do acesso e do URM, partindo do entendimento de que todas as atividades contribuem para este fim. Por esse motivo, todas as dimensões, subdimensões, indicadores e medidas do modelo avaliativo foram considerados como tendo o mesmo grau de importância (MARIN ET AL., 2003).

A matriz de julgamento apresentada no quadro 2 sugere critérios para a emissão do juízo de valor da gestão. Para facilitar a operacionalização da análise, cada designação recebeu os seguintes escores: 5 (cinco) para a classificação 'bom'; 3 (três) para 'regular'; 0 (zero) para 'ruim'. Tais escores possibilitam o cumprimento dos critérios de julgamento propostos, a partir da somatória, em cada componente. 
Quadro 2. Matriz de julgamento da gestão municipal na Purm. Brasil, 2015

\begin{tabular}{|c|c|c|c|c|}
\hline & \multirow{2}{*}{ Subdimensão } & \multicolumn{3}{|c|}{ Julgamento } \\
\hline & & Subdimensão & Dimensão & Gestão \\
\hline \multirow{3}{*}{ 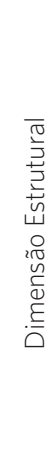 } & Recursos Humanos & $\begin{array}{l}\sum \text { dos escores: } \\
\text { 20-18: Bom (5); } \\
\text { 17-11: Regular (3); } \\
\text { 10-0: Ruim (0). }\end{array}$ & & \multirow{6}{*}{$\begin{array}{l}\text { } \sum \text { dos escores: } \\
\text { 10-8: Bom; } \\
\text { 7-6: Regular; } \\
\text { 5-0: Ruim. }\end{array}$} \\
\hline & Infraestrutura & $\begin{array}{l}\sum \text { dos escores: } \\
\text { 20-16: Bom (5); } \\
\text { 15-11: Regular (3); } \\
\text { 10-0: Ruim (0). }\end{array}$ & $\begin{array}{l}\sum \text { dos escores: } \\
\text { 15-13: Bom (5); } \\
\text { 12-6: Regular (3); } \\
\text { 5-0: Ruim (0). }\end{array}$ & \\
\hline & $\begin{array}{l}\text { Mecanismos de Decisão } \\
\text { e Controle }\end{array}$ & $\begin{array}{l}\sum \text { dos escores: } \\
\text { 25-21: Bom (5); } \\
\text { 20-11: Regular (3); } \\
\text { 10-0: Ruim (0). }\end{array}$ & & \\
\hline \multirow{3}{*}{ 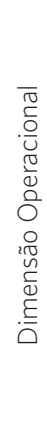 } & $\begin{array}{l}\text { Seleccão, Programação e } \\
\text { Aquisição }\end{array}$ & $\begin{array}{l}\sum \text { dos escores: } \\
\text { 30-24: Bom (5); } \\
\text { 23-16: Regular (3); } \\
\text { 15-0: Ruim (0). }\end{array}$ & \multirow{3}{*}{$\begin{array}{l}\sum \text { dos escores: } \\
\text { 15-13: Bom (5); } \\
\text { 12-6: Regular (3); } \\
\text { 5-0: Ruim (0). }\end{array}$} & \\
\hline & $\begin{array}{l}\text { Armazenamento e } \\
\text { Distribuição }\end{array}$ & $\begin{array}{l}\sum \text { dos escores: } \\
\text { 25-21: Bom (5); } \\
\text { 20-11: Regular (3); } \\
\text { 10-0: Ruim (0). }\end{array}$ & & \\
\hline & $\begin{array}{l}\text { Prescrição e Dispensa- } \\
\text { ção de Medicamentos }\end{array}$ & $\begin{array}{l}\sum \text { dos escores: } \\
\text { 20-15: Bom (5); } \\
\text { 10-0: Ruim (0). }\end{array}$ & & \\
\hline
\end{tabular}

Fonte: Elaboração própria.

\section{Conclusões}

A metodologia participativa adotada neste estudo resultou em um modelo avaliativo que possibilitou uma rica discussão a respeito do problema do uso inadequado de medicamentos, dos objetivos da Purm e dos resultados esperados com a intervenção, além dos processos necessários para alcançá-los, com vistas à análise da plausibilidade do modelo teórico, lógico e da matriz, propiciando a obtenção do consenso, entre os participantes, sobre os elementos.

O modelo avaliativo proposto foi considerado apropriado pelo grupo de especialistas, que julgaram todos os itens coerentes e passíveis de obtenção, indicando que o mesmo pode ser utilizado em posteriores estudos avaliativos. É importante ressaltar que pequenos ajustes no modelo teórico e lógico, assim como na matriz de avaliação, podem ser necessários no decorrer das aplicações do instrumento, e a qualquer momento podem ser revisados.

A preocupante situação mundial com o uso inadequado de medicamentos, a sua representação simbólica na sociedade, assim como as determinações nacionais sobre o tema e o contexto em que se dá a descentralização SUS conferem à gestão municipal um papel fundamental na Purm, junto aos profissionais de saúde e à comunidade. $\mathrm{O}$ desenvolvimento de um modelo avaliativo para o município pretende viabilizar a realização de avaliações situacionais, com a identificação dos fatores que possam contribuir para a tomada de decisões e a melhoria de resultados, passível de reaplicação periódica para o 
acompanhamento das ações desenvolvidas. $\mathrm{O}$ instrumento elaborado tem o propósito de ser indutor de reformulação de práticas e chamar a atenção dos gestores para as atividades de Purm, a importância e vantagens da sua execução, que vão desde o incremento efetivo na saúde da população ao racionamento dos recursos públicos.

\section{Referências}

BARRETO, J. L.; GUIMARÃES, M. C. L. Avaliação da gestão descentralizada da assistência farmacêutica básica em municípios baianos, Brasil. Cad. Saúde Pública, Rio de Janeiro, v. 26, n. 6, p. 1207-1220, jun. 2010.

BRASIL. Ministério da Saúde. Aquisição de medicamentos para a assistência farmacêutica no SUS: Orientações básicas. Brasília, DF: Ministério da Saúde, 2006.

Resolução nº 338, de 06 de maio de 2004. Diário Oficial [da] União, Brasília, DF, 2004. Disponível em: <http://bvsms.saude.gov.br/bvs/saudelegis/cns/2004/ res0338_06_05_2004.html>. Acesso em: 26 out. 2016.

Resolução - RDC n ${ }^{\circ}$ 36, de 25 de julho de 2013. Institui as ações para a segurança do paciente em serviços de saúde e dá outras providências. Diário Oficial [da] União, Brasília, DF, 2013a. Disponível em: <http://bvsms.saude.gov.br/bvs/saudelegis/ anvisa/2013/rdc0036_25_07_2013.html>. Acesso em: 26 out. 2016.

Portaria n 3.916, de 30 de outubro de 1998.

Diário Oficial [da] União, Brasília, DF, 1998. Disponível em: <http://bvsms.saude.gov.br/bvs/saudelegis/

\section{Agradecimentos}

À Coordenação de Aperfeiçoamento de Pessoal de Nível Superior (Capes), pela concessão da bolsa de mestrado durante todo o período de realização deste trabalho.

gm/1998/prt3916_30_10_1998.html>. Acesso em: 26 out. 2016.

Portaria $n^{\circ} 529$, de $1^{\circ}$ de abril de 2013. Institui o Programa Nacional de Segurança do Paciente (PNSP). Diário Oficial [da] União, Brasília, DF, 2013b. Disponível em: <http://bvsms.saude.gov.br/bvs/saudelegis/ gm/2013/prt0529_01_04_2013.html>. Acesso em: 26 out. 2016.

. Senado Federal. Constituição da República

Federativa do Brasil: Artigo 196. Brasília, DF, 1988.

CAPONI, S. et al. Medicalização da vida: ética, saúde pública e indústria farmacêutica. Palhoça: Unisul, 2010.

\section{CENTER FOR DISEASE CONTROL AND}

PREVENTION (CDC). Framework for program evaluation in public health. Mmwr, Atlanta, v. 48, n. 11, p. 1-58, set. 1999.

COELHO, H. L. L.; PINHEIRO, R. M.; MAGARINOS-

TORRES, R. Promoção do Uso Racional de Medicamentos. In: OSÓRIO-DE-CASTRO, C. G. S. et al. Assistência farmacêutica: gestão e prática para profissionais da saúde. Rio de Janeiro: Fiocruz, 2014. p. 283-294. 
CONSELHO FEDERAL DE FARMÁCIA (CFF). $A$

assistência farmacêutica no SUS. Brasília, DF: CFF, 2010.

CONTANDRIOPOULOS, A. P. et al. A avaliação na área da saúde: conceitos e métodos. In: HARTZ, Z. M. A. Avaliação em saúde. 2. ed. Rio de Janeiro: Fiocruz, 2002. p. 29-47.

EV, L. S.; GONÇALVES, C. B. C. Utilização de medicamentos. In: OSÓRIO-DE-CASTRO, C. G. S. et al. Assistência farmacêutica: gestão e prática para profissionais da saúde. Rio de Janeiro: Fiocruz, 2014. p. 119-134.

FERREIRA, R. C.; FIORINI, V. M. L.; CRIVELARO, E. Formação profissional no SUS: o papel da Atenção Básica em Saúde na perspectiva docente. Rev. Bras. Educ. Med., Rio de Janeiro, v. 34, n. 2, p. 207-215, jun. 2010.

IVAMA-BRUMMELL, A. M.; LYRA JÚNIOR, D.; SAKAI, M. H. Recursos Humanos para a Assistência Farmacêutica no Sistema Único de Saúde. In: OSÓRIODE-CASTRO, C. G. S. et al. Assistência farmacêutica: gestão e prática para profissionais da saúde. Rio de Janeiro: Fiocruz, 2014. p. 69-78.

LEITE, S. N.; VIEIRA, M.; VEBER, A. P. Estudo de utilização de medicamentos: uma síntese de artigos publicados no Brasil e na América Latina. Ciên. Saúde Coletiva, Rio de Janeiro, v. 13, supl., p. 793-802, abr. 2008.

LEVITON, L. C. et al. Evaluability assessment to improve public health policies, programs, and practices. Annual Review Of Public Health, Palo Alto, v. 31, n. 1, p. 213-233, 2010.

MARIN, N. et al. Assistência farmacêutica para gerentes municipais. Rio de Janeiro: Opas; OMS, 2003. 373 p.

MELO, D. O.; RIBEIRO, E.; STORPIRTIS, S. A importância e a história dos estudos de utilização de medicamentos. Revista Brasileira de Ciências
Farmacêuticas, São Paulo, v. 42, n. 4, p. 475-485, out./ dez. 2006.

MENDES, S. J. et al. Gestão da assistência farmacêutica: avaliação de um município catarinense. Revista Eletrônica Gestão \&̇ Saúde, Brasília, DF, v. 6, n. 1, p. 4-29, jan. 2015.

\section{NUNES, C. C.; AMADOR, T. A.; HEINECK, I. O}

medicamento na rotina de trabalho dos Agentes Comunitários de Saúde da Unidade Básica de Saúde Santa Cecília, em Porto Alegre, RS, Brasil. Saúde Soc., São Paulo, v. 17, n. 1, p. 85-94, 2008.

\section{PEPE, V. L. E.; OSÓRIO-DE-CASTRO, C. G. S. A} interação entre prescritores, dispensadores e pacientes: informação compartilhada como possível benefício terapêutico. Cad. Saúde Pública, Rio de Janeiro, v. 16, n. 3, p. 815-822, set. 2000.

PINTO JÚNIOR, E. P. et al. Análise da produção científica sobre avaliação, no contexto da estratégia e saúde da família, em periódicos brasileiros. Saúde em Debate, Rio de Janeiro, v. 39, n. 104, p. 268-278, jan./ mar. 2015.

SOUZA, L. E. P. F.; SILVA, L. M. V.; HARTZ, Z. M. A. Conferência de consenso sobre o objeto-imagem da descentralização da atenção à saúde no Brasil. In: HARTZ, Z. M. A.; SILVA, L. M. V. Avaliação em saúde: dos modelos teóricos à prática na avaliação de programas e sistemas de saúde. Rio de Janeiro: Fiocruz, 2005. p. 65-91.

\section{WORLD HEALTH ORGANIZATION (WHO).}

Promoting rational use of medicine: core components, 2002. Disponível em: <http://apps.who.int/ medicinedocs/pdf/h301le/h301le.pdf >. Acesso em: 15 abr. 2014.

\footnotetext{
Recebido para publicação em abril de 2016

Versão final em setembro de 2016

Conflito de interesses: inexistente

Suporte financeiro: não houve
} 